process (in terms of survival probability). This notion is supported by the spline analysis associating remoteness of the cancer diagnosis with better outcomes as well as the finding that patients with a cancer history were older and had more comorbidities than their counterparts. Statistical modeling can hardly make up for that difference between the 2 groups. Further, cause-specific mortality is often difficult to study. For one, adjudication of the cause of death is often insufficient, and misclassification is common (eg, missed cancer in the control group vs biased adjudication due to previous cancer history). Notably, the authors do not account for cancer diagnosed after $\mathrm{CABG}$, and therefore the comparison should not be seen as one strictly comparing subjects with and without cancer altogether. Eventually, the combined proportion of CVD- and cancerdeath amount to almost the same in both groups $(78 \%$ vs $72 \%$ ), as a substantial proportion of patients also develop cancer during follow up (undisclosed) and die a cancer death during follow-up (18\%).

A final word of caution concerns the competing risk analysis with subhazard estimation. The authors find a significantly increased subhazard for cancer death in patients with a history of cancer but also a significantly lower subhazard for cardiovascular mortality. A causal interpretation of this would be that a cancer history is protective against dying a cardiovascular death. Substantial literature would suggest otherwise. ${ }^{4}$ Rather, the explanation here is given by a peculiarity of this popular methodology (Fine and Gray competing risk models), which was not actually devised for causal interpretations. To estimate the subhazards, an unnatural assumption that subjects remain part of the risk set, after a competing event has occurred, must be made. Consequently, any variable that increases the cause-specific risk of event A will appear to decrease the subdistribution hazard for event B. Because whenever a type A event occurs, it eliminates the possibility that a type B event will happen. We commend the authors for presenting this large cohort study with some important findings but also caution against a conclusion that suggests cardiovascular prognosis is the same for patient with and without a cancer.

\section{References}

1. Koene RJ, Prizment AE, Blaes A, Konety SH. Shared risk factors in cardiovascular disease and cancer. Circulation. 2016;133:1104-14.

2. Zaorsky NG, Churilla TM, Egleston BL, Fisher SG, Ridge JA, Horwitz EM, et al. Causes of death among cancer patients. Ann Oncol. 2017;28:400-7.

3. Mennander A, Nielsen SJ, Huhtala H, Dellgren G, Hansson EC, Jeppsson A. History of cancer and survival after coronary artery bypass grafting: experience from the SWEDEHEART registry. J Thorac Cardiovasc Surg. 2022;164:107-14.e1.

4. Sturgeon KM, Deng L, Bluethmann SM, Zhou S, Trifiletti DM, Jiang C, et al. A population-based study of cardiovascular disease mortality risk in US cancer patients. Eur Heart J. 2019;40:3889-97.

\title{
Commentary: Are all cancers equal?
}

\section{Sigrid E. Sandner, MD, ${ }^{\mathrm{a}}$ and \\ Mario Gaudino, MD, MSCE ${ }^{\mathrm{b}}$}

Cardiovascular disease and cancer are the 2 leading causes of death in the United States to date. In 2018, in the United States, the age-adjusted death rate from cardiovascular

\footnotetext{
From the ${ }^{\mathrm{a}}$ Department of Cardiac Surgery, Medical University of Vienna, Austria; and ${ }^{b}$ Department of Cardiothoracic Surgery, Weill Cornell Medicine, New York Presbyterian Hospital, New York, NY.

Disclosures: The authors reported no conflicts of interest.

The Journal policy requires editors and reviewers to disclose conflicts of interest and to decline handling or reviewing manuscripts for which they may have a conflict of interest. The editors and reviewers of this article have no conflicts of interest.

Received for publication Oct 6, 2020; revisions received Oct 6, 2020; accepted for publication Oct 7, 2020; available ahead of print Oct 17, 2020.

Address for reprints: Mario Gaudino, MD, MSCE, Department of Cardiothoracic Surgery, Weill Cornell Medicine, New York Presbyterian Hospital, 525 East 68th St,

New York, NY 10065 (E-mail: mfg9004@med.cornell.edu).

J Thorac Cardiovasc Surg 2022;164:116-8

0022-5223/\$36.00

Copyright (c) 2020 by The American Association for Thoracic Surgery

https://doi.org/10.1016/j.jtcvs.2020.10.030
}

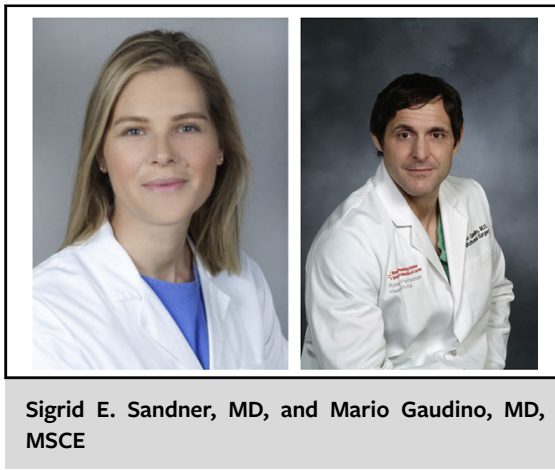

CENTRAL MESSAGE

Long-term cardiovascular and

cancer-related mortality are

important outcomes after CABG

in patients with coexistent cancer

but may differ with respect to

cancer type. 
disease and cancer was 163.6 and 149.1 deaths per 100,000 population, respectively. ${ }^{1}$ Cancer survivors are at increased risk for cardiovascular disease compared with the general population. ${ }^{2}$ This is likely due to shared risk factors for both diseases in an increasingly aging population, as well as cardiovascular toxicities of anticancer therapies. ${ }^{3}$ More than 1 in 10 patients with cancer do not die from their cancer but from cardiovascular disease. ${ }^{3}$ For some cancers, like breast, prostate, endometrial, and thyroid cancer, around one-half will die from cardiovascular disease.

Surgeons have generally been reluctant to accept patients with coexistent cancer for coronary revascularization, due to reduced life expectancy with various cancer subtypes, and the increased risk of postoperative complications due to cancer-associated thrombotic and/or bleeding risk. Previous analyses that have studied the prognostic impact of cancer on coronary artery bypass grafting $(\mathrm{CABG})$ outcomes have been reported from single centers limited by small sample sizes. ${ }^{4}$ Most recently, an analysis of the U.S. National Inpatient Sample of $>2$ million hospitalizations for CABG including $>180,000$ patients with comorbid cancer showed that, apart from increased major bleeding with breast or prostate cancer, patients with coexistent cancer had similar in-hospital outcomes with those without cancer, suggesting that CABG should not be declined in patients with cancer when otherwise indicated.

In this issue of the Journal, Mennander and colleauges ${ }^{6}$ report the long-term mortality of 82,137 patients undergoing CABG between 1997 and 2015, of which 6819 procedures $(8.3 \%)$ were performed in patients with a history of cancer, with the proportion increasing over time from $3.8 \%$ in 1997 to $14.8 \%$ in 2015 . The analysis draws on the extensive dataset of the SWEDEHEART registry which, with its large number of participants and clinical variables collected for each patient, and near-completeness of follow-up, provides the unique opportunity to analyze longitudinal data and generate generalizable evidence on the outcomes of patients undergoing CABG. The most commonly encountered cancers were prostate cancer $(31 \%)$, followed by gastrointestinal cancer $(12 \%)$, cancer of the kidneys $(11 \%)$, and breast cancer $(8 \%)$. Patients with a history of cancer were more likely to have prevalent comorbid conditions that are associated with adverse outcomes. The authors found an increased adjusted long-term mortality risk (hazard ratio, 1.33; $95 \%$ confidence interval, 1.28-1.38) in patients with a history of cancer that was driven by cancer-related mortality and was inversely associated with the time interval from cancer diagnosis to CABG. Cardiovascular mortality was comparable in patients with a history of cancer and those without cancer undergoing CABG (hazard ratio, 1.02; 95\% confidence interval, 0.96-1.08).

The choice to revascularize, and the choice of revascularization strategy, in patients with coexistent cancer is challenging, with little evidence to guide clinical decision-making. The authors conclude that CABG may be recommended to patients with a history of cancer and a reasonable life expectancy. Herein, however, arguably also lies the selection bias confounding this analysis. It is highly unlikely that patients who do not meet the ill-defined term of "reasonable" lifeexpectancy would have been referred for CABG, and thus such patients remain excluded from analysis.

In addition, the SWEDEHEART registry does not distinguish between current and historical cancer at the time of CABG. This is particularly relevant, as the prognostic impact of coexistent cancer in patients after percutaneous coronary intervention has been shown to be specific both for the type of cancer and the presence of metastases, and whether the diagnosis of cancer was current or historical. ${ }^{7}$ The authors calculate a cut-off point of 2.5 years for the time period between cancer diagnosis and CABG beyond which history of cancer was not significantly associated with all-cause mortality. Whether this finding can be factored into clinical decision-making requires further study, as such a time period may differ widely for various cancer subtypes. In an analysis of 4079 patients of whom $82(2.1 \%)$ had coexistent solid-organ cancer, Garatti and colleagies ${ }^{8}$ showed that the 5 - and 10-year survival rates after CABG were significantly lower in patients with cancer, with major differences between cancer subtypes. Whereas survival after $\mathrm{CABG}$ in patients with breast cancer is $86 \%$ at 5 years, it is a dismal $32 \%$ in those with lung cancer. Undoubtedly, future reports out of the SWEDEHEART registry will also provide an analysis of survival with regard to types of cancer.

The authors are commended for adding valuable information on the long-term outcomes of CABG in a population of patients with a comorbid condition whose prevalence increased by $289 \%$ over a period of 18 years. It is reassuring that following surgical revascularization their cardiovascular mortality risk is comparable with that of the cancer-free population. Nevertheless, with many unknowns remaining, the decision to revascularize must still be made individually for each patient, based on cancer type, historical or current cancer diagnosis, and careful evaluation of cancer-related and cardiovascular mortality risk.

\section{References}

1. Centers for Disease Control and Prevention. Mortality in the United States, 2018 NCHS Data Brief. 2020 (No. 355)Available at: https://www.cdc.gov/nchs/data/ databriefs/db355-h.pdf. Accessed October 5, 2020.

2. Strongman H, Gadd S, Matthews A, Mansfield KE, Stanway S, Lyon AR, et al Medium and long-term risks of specific cardiovascular diseases in survivors of 20 adult cancers: a population-based cohort study using multiple linked UK electronic health records databases. Lancet. 2019;394:1041-54.

3. Sturgeon KM, Deng L, Bluethmann SM, Zhou S, Trifiletti DM, Jiang C, et al. A population-based study of cardiovascular disease mortality risk in US cancer patients. Eur Heart J. 2019;40:3889-97.

4. Kucukarslan N, Tatar T, Uzun M, Yavuz I, Ozal E, Tatar H. Coronary artery bypass surgery in patients with malignancy: a single-center study with comparison to patients without malignancy. J Card Surg. 2009;24:151-5. 
5. Guha A, Dey AK, Kalra A, Gumina R, Lustberg M, Lavie CJ, et al. Coronary artery bypass grafting in cancer patients: prevalence and outcomes in the United States. Mayo Clin Proc. 2020;95:1865-76.

6. Mennander A, Nielsen SJ, Huhtala H, Dellgren G, Hansson EC, Jeppsson A. History of cancer and survival after coronary artery bypass grafting: experiences from the SWEDEHEART Registry. J Thorac Cardiovasc Surg. 2022;164:107-14.e1.
7. Potts JE, Iliescu CA, Lopez Mattei JC, Martinez SC, Holmvang L, Ludman P, et al. Percutaneous coronary intervention in cancer patients: a report of the prevalence and outcomes in the United States. Eur Heart J. 2019;40:1790-800.

8. Garatti A, D’Ovidio M, Saitto G, Daprati A, Canziani A, Mossuto E, et al. Coronary artery bypass grafting in patients with concomitant solid tumours: early and long-term results. Eur J Cardiothorac Surg. 2020;58:528-36. 